\title{
Role of Resection of the Primary Pancreatic Neuroendocrine Tumour Only in Patients with Unresectable Metastatic Liver Disease: A Systematic Review
}

\author{
Gabriele Capurso $^{a}$ Rossella Bettini ${ }^{b}$ Maria Rinzivillo ${ }^{a}$ Letizia Boninsegna ${ }^{b, c}$ \\ Gianfranco Delle Fave ${ }^{a}$ Massimo Falconi ${ }^{b}$ \\ a Digestive and Liver Disease Unit, II School of Medicine, 'Sapienza' University of Rome, Rome, \\ ${ }^{\mathrm{b}}$ Department of Surgery, Ospedale Sacro Cuore-Don Calabria, Negrar, and 'Department of Surgery, \\ University of Verona, Verona, Italy
}

\section{Key Words}

Pancreatic neuroendocrine tumours · Surgery •

Metastatic disease $\cdot$ Systematic review

\begin{abstract}
Background: Surgery remains the only curative option for pancreatic neuroendocrine tumours (PNETs), but its indication is limited by metastatic disease in most patients. Indication for removing the primary lesion only in the setting of unresectable liver disease is controversial. The present systematic review aims at determining the potential benefits (survival, progression-free survival) or harms (morbidity, mortality) of surgical resection of the primary lesion only in patients with PNETs and unresectable metastases. Methods: Medline was queried for studies reporting the outcome of PNET patients with unresectable liver metastases whenever there was an explicit comparison between resection of the primary lesion only ('active treatment') and no resection ('non-active treatment'). The primary outcome was survival; possible secondary outcomes were progression-free survival, treatment-related mortality and morbidity, and relief of symptoms. Results: Only 3 cohort studies found were eligible and analysed; no meta-analysis could be performed. The number of patients undergoing 'active treatment' varied
\end{abstract}

\section{KARGER}

Fax +4161306 1234

E-Mail karger@karger.ch

www.karger.com
(C) 2011 S. Karger AG, Basel

0028-3835/11/0934-0223\$38.00/0

Accessible online at:

www.karger.com/nen from 16 to 20 , with a percentage ranging from 17 to $39 \%$ of cohorts. Survival was longer in patients who received 'active treatment' in 2 studies, and the 5 -year survival rate also seemed higher, without significant complications. Discussion: Available data suggest a possible benefit of resection of the primary lesion only in this setting. However, a bias towards a more aggressive surgical approach in patients with a better performance status or less advanced disease seems likely, and no conclusion can be drawn except for the need of randomised trials. We calculated that such a trial would require at least 118 patients per arm.

Copyright $\odot 2011$ S. Karger AG, Basel

\section{Introduction}

Pancreatic neuroendocrine tumours (PNETs) are heterogeneous neoplasms which are generally considered rare. However, their incidence has almost doubled in the last 20 years [1]. Although PNETs represent only $\sim 1 \%$ of all pancreatic neoplasms by incidence, their prevalence is close to $10 \%$ [2], likely due to their relatively 'indolent' clinical course. PNETs are challenging tumours and generally display worse prognosis compared to neuroendocrine tumours (NETs) arising from the gastrointestinal 
tract [3]. Surgery remains the only curative therapeutic approach for PNETs to date, but its indication is often limited by the fact that most PNETs present with metastatic disease at diagnosis.

Nevertheless, as most PNETs are characterised by a relatively low aggressive nature and slow growth even in the setting of advanced disease with the 5-year survival exceeding $60 \%$ [4], a tendency to promote aggressive surgery, even in the presence of liver metastases, exists. This aggressive surgical approach seems justified whenever radical resection of both the primary tumour and the liver metastases is achievable. However, at the time of diagnosis, more than $80 \%$ of the liver metastases are bilobar [5], and curative resection is not possible. On the other hand, in patients not suitable for radical resection due to local vascular infiltration or liver metastases extending to both liver lobes, the indication for surgery remains highly controversial [6].

Cytoreductive surgery, also termed debulking, refers to the greatest possible reduction of the tumour mass in the context of the persistence of metastases [7] and is generally carried out when it is possible to remove $70-90 \%$ of the disease.

In the absence of extrahepatic disease, synchrony liver and pancreatic resection is feasible and seems to increase survival also if the recurrence rate approaches $80 \%$, and the risk of such a major surgery has to be considered [810]. The indication to remove the primary PNET only in the setting of unresectable liver metastases is even more debated. The potential benefits of this approach may be to provide relief from hormonal and local tumour-related symptoms and to increase survival improving the efficacy of further treatment by decreasing overall tumour burden and limiting the disease to the liver.

Some authors have proposed this aggressive strategy [11] but literature data are difficult to evaluate as they often refer to heterogeneous series both in terms of tumour features, such as primary site and associated syndrome (functioning and non-functioning), and of surgical strategy, as data on patients treated with debulking of the primary PNET only either in the presence of metastatic or locally advanced disease are often mixed with those of patients treated with both pancreatic and hepatic debulking.

We therefore designed a systematic review aimed at determining the potential benefits or harms of surgical resection of the primary lesion, without concomitant resection of metastases, in patients with PNETs and unresectable liver metastases as compared to other treatments not including surgery.

\section{Methods}

\section{Search Strategy}

A computerised literature search of Medline and the Cochrane database of systematic reviews for a prior systematic review concerning the selected topic revealed no published papers. In the present search for original studies, Medline was queried until August 2009 using the following search terms: (neuroendocrine tumor OR neuroendocrine tumors OR neuroendocrine tumour OR neuroendocrine tumours OR adenoma OR adenomas OR apudoma OR Apudomas OR Carcinoma, Islet Cell OR Gastrinoma OR Glucagonoma OR Insulinoma, OR Adenoma, Islet Cell OR Somatostatinoma OR Vipoma OR beta-Cell Tumor OR Tumors, Island Cell OR Islet Cell Tumors OR Tumor, Island Cell OR Tumor, Islet Cell OR Islet Cell Adenoma OR Islet Cell Carcinoma OR Pancreatic Endocrine Tumour OR Islet Cell Tumor, Ulcerogenic) AND (Operative Surgical Procedure OR Operative Surgical Procedures OR Procedures, Operative Surgical OR Surgical Procedure, Operative OR Operative Procedures OR Operative Procedure OR Procedure, Operative OR Procedures, Operative OR Procedure, Operative Surgical OR Pancreatectomies OR Pancreaticoduodenectomy OR Pancreatoduodenectomies OR Duodenopancreatectomy OR Duodenopancreatectomies OR Primary Resection OR Splenopancreasectomy OR Primary Tumour Resection) AND (metastatic OR metastasis OR secondary OR spread OR advanced OR Metastases, Neoplasm OR Neoplasm Metastases OR Metastasis OR Metastases OR Metastasis, Neoplasm OR Residual Neoplasm OR Neoplasms, Residual OR Residual Neoplasms OR Residual Cancer OR Cancer, Residual OR Cancers, Residual OR Residual Cancers OR Residual Tumor OR Residual Tumors OR Tumor, Residual OR Tumors, Residual) AND (liver OR hepatic).

To identify additional studies, the references of all the identified papers for further relevant articles were reviewed. The titles of all identified articles were screened to ascertain their relevance. Abstracts and/or full texts of selected potentially relevant papers were further evaluated.

\section{Criteria for Considering Studies for the Review}

Only studies reporting the outcome of patients with PNETs and no radically resectable liver metastases (irrespective of presence or absence of extrahepatic disease, tumour grade or functional status) were considered. We established to consider for inclusion different study types, irrespective of language, publication status, or sample size, with the following hierarchy. First, we aimed at analysing randomised controlled trials, quasi-randomised controlled trials and non-randomised controlled trials. However, given the likely paucity of high-quality data on this topic, prospective or retrospective cohort studies were also considered for the review. They were included whenever there was an explicit comparison between the following two treatments arms: (1) resection of the primary pancreatic lesion only, in the presence of unresectable liver metastases ('active treatment'); (2) no resection of the primary pancreatic lesion in the presence of unresectable liver metastases ('non-active treatment'). In both arms other medical (somatostatin analogues, chemotherapy, peptide receptor radiotherapy) or ablative (trans-arterial chemoembolisation, radiofrequency ablation, cryoablation) treatments were permitted. 'Controlled before-and-after studies', historically controlled trials, case-control studies, case reports or case series were not 
Fig. 1. Results of search and reasons for exclusion of papers according to QUORUM statement.
Potentially relevant studies identified and screened for retrieval $(n=3,108)$

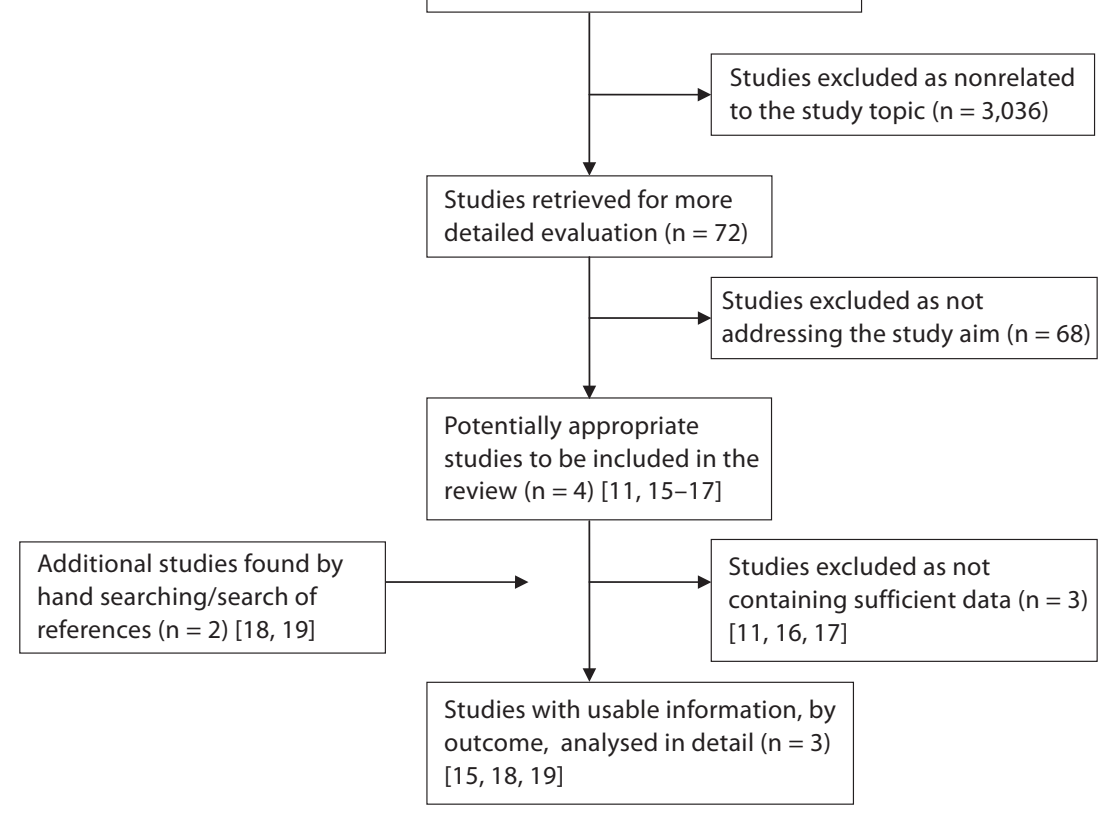

included. In case of duplicate publications, the most recent or more complete publication was used.

Two independent reviewers (G.C., M.R.) carried out study identification, selection and data extraction, and discussed disagreements with a third reviewer (M.F.). Excluded studies and the reasons for exclusion were recorded.

\section{Measured Outcomes}

The primary evaluated outcome was the survival, expressed as proportion of patients alive after $1,3,5$, or 10 years from the intervention, and/or as median survival in the group. Possible secondary outcomes were the estimated progression-free survival, treatment-related mortality, treatment-related morbidity (surgery - 30-day mortality, bile leak, lymphorrhoea, abdominal collections requiring treatment, wound-related complications, such as wound infection, wound dehiscence), relief of symptoms (however defined by authors; hormonal symptom, mass symptoms: biliary obstruction, gastric outlet obstruction, gastrointestinal haemorrhage). Finally, if feasible, potential factors predicting a better outcome such as hepatic tumour burden, grading or other tumour features were evaluated.

\section{Data Extraction}

From the studies that met the eligibility criteria the following data were collected: (1) study: publication year, study design, study location; (2) cases: definition (i.e. clinical charts or histological diagnosis or other means), number and source of cases, accrual period; (3) intervention: type of surgery in the 'active' treatment \pm additional treatments; type of 'non-surgical' inter- ventions in the 'non-active treatment'; (4) possible type of covariates considered: sex, age, race, body mass index, tumour features; (5) considered outcomes; (6) other possible factors influencing outcomes; (7) duration of follow-up; (8) possible percentage of individuals refusing to undergo a treatment; (9) outcome measure expressed as median (or mean) survival, and/or survival at different time courses; (10) possible treatment-related mortality or morbidity, and (11) possible relief of symptoms.

\section{Statistical Analysis}

A meta-analysis of the possibly identified randomised clinical trials using the software package MedCalc was planned with the following methods: for dichotomous variables, calculation of the relative risk with $95 \%$ confidence interval for continuous variables calculation of the mean difference; use of a random-effects model [12], evaluation of the heterogeneity by $\chi^{2}$ test, and measure of the quantity of heterogeneity by $\mathrm{I}^{2}[13]$, considering an $\mathrm{I}^{2}$ of $30 \%$ or more to represent heterogeneity. It was also planned to evaluate possible biases applying a funnel plot [14].

\section{Results}

\section{Search Result and Study Selection}

As shown in figure 1, a total of 3,108 references were identified through the Medline search. 3,036 studies were excluded as non-related to the study topic. The abstracts 
Table 1. Summary of the 6 studies initially considered potentially appropriate for the review

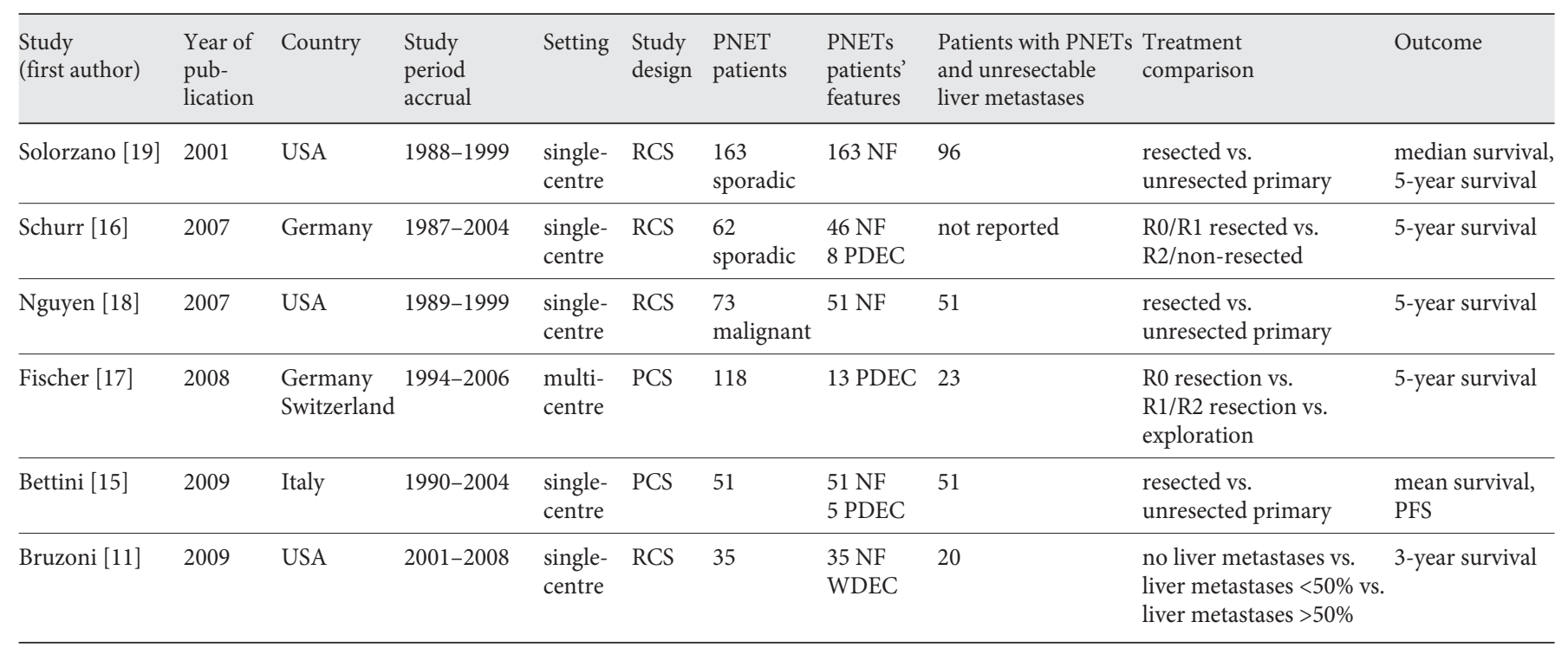

RCS = Retrospective cohort study; PCS = prospective cohort study; PFS = progression-free survival; NF = no-functioning; WDEC = well-differentiated endocrine carcinoma; $\mathrm{PDEC}=$ poorly differentiated endocrine carcinoma.

and/or full texts of 72 studies were examined in more detail, but 68 of them were further excluded, as - although dealing with endocrine tumours - they did not address the topic of the study. Of the 4 studies potentially appropriate to be included in the review [11, 15-17], 3 were excluded because they did not contain sufficient data for the two treatment arms $[11,16,17]$. Two additional studies were found by hand searching/search of references of the identified papers $[18,19]$, leaving 3 studies with usable information, by outcome, analysed in detail $[15,18,19]$. Agreement for these studies was $100 \%$ among the two reviewers. Unfortunately, none of the identified studies were randomised clinical trials, quasi-randomised trials, or non-randomised controlled trials. The 3 identified studies were analysed and data extracted to try and highlight possible useful information, although no formal meta-analysis could be performed.

\section{Study Characteristics}

Table 1 shows the descriptive characteristics of the 3 included studies and of 3 studies which were excluded at the final screening -4 of them were retrospective and 2 prospective cohort studies. Three of them took place in the USA and 3 in Europe, and only 1 was multicentric. The number of patients with PNETs and unresectable liver metastases varied from 20 to 96 and in one publication this number was not exactly stated. As stated above, 3 studies met the criteria of an explicit comparison between the two treatments: resection of the primary pancreatic lesion only, in the presence of unresectable liver metastases (i.e. 'active treatment') as compared to no resection of the primary pancreatic lesion in the presence of unresectable liver metastases (i.e. 'non-active treatment').

As shown in table 2, the number of patients undergoing the 'active treatment' varied from 16 to 20 , with a percentage ranging from 17 to $39 \%$ in the 3 studies. Two of the studies $[18,19]$ provided little or no information about patients' features according to the received treatment. In the third study [15], there were no differences between patients receiving 'active' or 'non-active' treatment as far as sex, age and tumour features, except for the percentage of patients with extensive liver involvement, which was higher in patients not receiving surgery (22 vs. 6\%). Interestingly, some two thirds of patients receiving surgery had their primary tumour in the body or tail of the pancreas in the 2 studies providing these data $[15,19]$, possibly suggesting a bias towards a preference for resection for tumours located in the body or tail.

\section{Survival Analysis}

As shown in table 3, the overall survival data for the two intervention groups were not provided in one of the studies [18], and did not differ significantly in the other 2 
Table 2. Characteristics of the patients in the 3 studies considered eligible for the review

\begin{tabular}{|c|c|c|c|c|c|c|}
\hline $\begin{array}{l}\text { Study } \\
\text { (first author) }\end{array}$ & $\begin{array}{l}\text { PNET patients } \\
\text { with unresectable } \\
\text { liver metastases }\end{array}$ & $\begin{array}{l}\text { Age } \\
\text { years }\end{array}$ & $\begin{array}{l}\text { Sex } \\
\% \text { male }\end{array}$ & $\begin{array}{l}\text { Median length of } \\
\text { follow-up, months }\end{array}$ & $\begin{array}{l}\text { Resected } \\
\text { primary, n (\%) }\end{array}$ & $\begin{array}{l}\text { Unresected } \\
\text { primary, n (\%) }\end{array}$ \\
\hline Nguyen [18] & 51 & not reported & 50.6 & 41 (range 2-192) & $20(39)$ & $31(61)$ \\
\hline Solorzano [19] & 96 & not reported & not reported & not reported & $16(17)$ & $80(83)$ \\
\hline
\end{tabular}

Table 3. Survival and symptomatic improvement in relation with the 'active' (resected) or 'not active' (unresected) group

\begin{tabular}{lllll}
\hline $\begin{array}{l}\text { Study } \\
\text { (first author) }\end{array}$ & $\begin{array}{l}\text { Median overall } \\
\text { survival, months }\end{array}$ & $\begin{array}{l}\text { 5-Year } \\
\text { survival, \% }\end{array}$ & $\begin{array}{l}\text { Median PFS } \\
\text { months }\end{array}$ & $\begin{array}{l}\text { Symptom } \\
\text { improvement }\end{array}$ \\
\hline $\begin{array}{c}\text { Bettini [15] } \\
\text { Resected }\end{array}$ & $54.3(95 \%$ CI 25-86) & 40.4 & $7.6(95 \%$ CI 0.5-14.7) & $88 \%$ \\
$\quad \begin{array}{l}\text { Unresected } \\
\text { Nguyen [18] }\end{array}$ & $39.5(95 \%$ CI 5.4-73.6) & 41.8 & $12(95 \%$ CI 3.7-20.3) & $31 \%$ \\
$\quad \begin{array}{l}\text { Resected } \\
\text { Unresected }\end{array}$ & not reported & 60 & not reported & not reported \\
Solorzano [19] & not reported & 30 & not reported & not reported \\
$\quad \begin{array}{l}\text { Resected } \\
\text { Unresected }\end{array}$ & $36(95 \%$ CI 26.4-96) & 49 & not reported & not reported \\
\hline
\end{tabular}

studies, although in both cases survival was longer in patients who had their primary tumour resected, with a median of 54.3 versus 39.5 months in the study by Bettini et al. [15], and of 36 versus 21.6 months in the study by Solorzano et al. [19]. The 5-year survival rate also seemed generally higher in patients who had their primary tumour resected, with a difference of $30 \%(60-30 \%)$ in one study (17), of 33\% (49-16\%) in a second (18), and no difference ( 40 vs. $41 \%$ ) in the study by Bettini et al. [15].

\section{Secondary Outcomes}

The progression-free survival data were available in one study only [15], and no significant difference was found. With regard to complications in the 'active treatment' group, there were no deaths related to surgery in 2 studies $[15,19]$, while no data specific for the subgroup of interest were given in the third study [18]. Similarly, the rate of complications and the need of reintervention seemed rather low, although these data were often lacking. With regard to symptomatic improvement, in the study by Bettini et al. [15] there was a clear trend towards a better control of symptoms in patients receiving surgery ( 88 vs. $31 \%$ ), while sufficient data were not provided in the other 2 studies.

Resection of the Primary Lesion Only in Metastatic PNETs

\section{Discussion}

The aim of the present study was to determine, throughout a systematic literature review, the potential benefits or harms of surgical resection of the primary lesion without concomitant resection of metastases, in patients with pancreatic endocrine tumours and unresectable liver metastases as compared to other treatments not including surgery.

Unfortunately, neither randomised nor quasi-randomised clinical trials evaluated this issue, and only 3 relatively small prospective [15] or retrospective $[18,19]$ cohort studies could be analysed in order to compare the 'active' surgical treatments to a 'non-active' treatment. In these 3 studies a group of patients ranging from 17 to $39 \%$ received surgery for the primary pancreatic lesion in the setting of advanced metastatic disease in the liver which was not treated surgically. Overall survival seemed longer in patients treated surgically (table 3), although our results are descriptive only, and no formal analysis has been possible. However, given the absence of randomisation in the examined studies, a bias towards a more aggressive surgical approach ('active treatment') in patients with a better overall performance status, a less advanced dis- 
ease, or possibly with location of the tumour in the body or tail of the pancreas seems likely, and no conclusion on this topic can be reasonably drawn except for the need of randomised trials.

One of our secondary aims was to identify potential factors predicting a better outcome after primary tumour resection. However, the identified studies did not contain sufficient information to permit such an analysis. Nevertheless, it is likely that factors such as histological features and primary tumour location within the pancreas could influence the outcome, with fewer complications and possibly a better outcome for patients receiving surgery for a tumour in the body or tail.

The issue of a possible indication for primary tumour removal in patients with gastroenteropancreatic endocrine tumours and unresectable liver metastases is relevant, as the majority of patients with NET, including these with PNETs, present with liver metastases at diagnosis, and a curative surgery is not feasible in most of them [4, 20,21]. However, published recommendations differ for NETs of the small intestine (carcinoids) and PNETs. Indeed, while ENETS (European Neuroendocrine Tumour Society) suggests the removal of the primary tumour to make liver metastases the only persisting problem for NETs of the jejunum and ileum [22], the resection of the primary tumour in metastatic PNETs is not recommended, except for selected low-risk patients with life-threatening symptoms due to complications of the primary lesion [23].

This recommendation is based not only on the higher rate of surgical complications for pancreatic compared to intestinal surgery, but also on data suggesting a better prognosis for patients with small intestine NETs and resected primary lesion even in the presence of residual metastatic disease in the liver. Indeed, some authors [24, 25] have reported that in the setting of metastatic disease, patients with a primary NET in the small intestine removed have a longer survival when compared to patients without resection of the primary tumour, although these few studies are retrospective and no randomised trials have been published.

The paucity of available data and the heterogeneity of series are a major problem limiting the possibility of an 'evidence-based' approach for gastroenteropancreatic endocrine tumours. There have been few attempts to perform systematic reviews in this specific field, and when attempts were made the results were disappointing as few conclusions could be drawn in the absence of randomised trials $[26,27]$.

Our review highlights the possible benefits of an aggressive surgical approach in metastatic PNETs, but also shows the limits of the available data, which include possible treatment bias as discussed above and the relatively low number of patients. Table 3 shows that there was a difference between the 5 -year survival rate between the 'active' and 'non-active' treatment of around $30 \%$ in 2 of the 3 studies examined $[18,19]$ and no difference in the third [15], in which, however, there was a trend for a better overall survival in patients receiving surgery. If one hypothesises that these differences may be real, a randomised trial with an $\alpha$-error of 0.10 and a $\beta$-error of 0.05 would need to enrol at least 50 patients per arm to make the possible $30 \%$ difference evident. If this difference is slightly lower, such as $25 \%$, a similar hypothesis would require at least 118 patients per arm. Given the relatively low incidence of PNETs, it is therefore evident that a randomised trial with adequate statistical power would only be possible in a multicentric setting. Such a trial should also take in account a number of factors relevant for the prognosis of these patients such as the spread of the hepatic and extrahepatic disease [28], the histological features including tumour differentiation and proliferation index [29], and the tumour site. On the other hand, the feasibility of this trial may be hampered by a number of clinically relevant practical obstacles, such as the possibility to treat these patients with other surgical or medical treatments available in the future, and the differences in available treatments for the remaining liver metastases for patients previously receiving pancreaticoduodenectomy. Therefore, it is likely that the hypothesised power calculation may not even be sufficient due to common protocol violations.

In the absence of other data, the resection of the primary tumour only for patients with PNETs and unresectable metastatic liver disease should only be considered in high-volume referral centres, with strict selection criteria and in a multidisciplinary setting of patients' care, and such procedures, apart from those dictated by the need to resolve life-threatening symptoms, belong to an experimental setting.

\footnotetext{
References $\quad>_{1}$ Fitzgerald TL, Hickner ZJ, Schmitz M, Kort EJ: Changing incidence of pancreatic neoplasms. A 16-year review of statewide tumor registry. Pancreas 2008;37:134-138.

-2 Yao JC, Eisner MP, Leary C, Dagohoy C, Phan A, Rashid A, Hassan M, Evans DB: Population-based study of islet cell carcinoma. Ann Surg Oncol 2007;14:3492-3500.

- 3 Metz DC, Jensen RT: Gastrointestinal neuroendocrine tumors: pancreatic endocrine tumors. Gastroenterology 2008; 135:14691492.
} 
-4 Panzuto F, Nasoni S, Falconi M, Corleto VD, Capurso G, Cassetta S, Di Fonzo M, Tornatore V, Milione M, Angeletti S, Cattaruzza MS, Ziparo V, Bordi C, Pederzoli P, Delle Fave G: Prognostic factors and survival in endocrine tumor patients: comparison between gastrointestinal and pancreatic localization. Endocr Relat Cancer 2005;12:10831092.

5 Chamberlain RS, Canes D, Brown KT, Saltz L, Jarnagin W, Fong Y, Blumgart LH: Hepatic neuroendocrine metastases: does intervention alter outcomes? J Am Coll Surg 2000; 190:432-445.

6 Hodul PJ, Strosberg JR, Kvols LK: Aggressive surgical resection in the management of pancreatic neuroendocrine tumors: when is it indicated? Cancer Control 2008;15:314321.

7 Wong RJ, DeCosse JJ: Cytoreductive surgery. Surg Gynecol Obstet 1990;170:276-281.

8 Norton JA, Warren RS, Kelly MG, Zuraek $\mathrm{MB}$, Jensen RT: Aggressive surgery for metastatic liver neuroendocrine tumors. Surgery 2003;134:1057-1065.

-9 Sarmiento JM, Que FG, Grant CS, Thompson GB, Farnell MB, Nagorney DM: Concurrent resections of pancreatic islet cell cancers with synchronous hepatic metastases: outcomes of an aggressive approach. Surgery 2002;132:976-983.

-10 Scigliano S, Lebtahi R, Mairie F, Stievenart JL, Kianmanesh R, Sauvanet A, Vullierme MP, Couvelard A, Belghiti J, Ruszniewski P, Le Guludec D: Clinical and imaging followup after exhaustive liver resection of endocrine metastases: a 15-year monocentric experience. Endocr Relat Cancer 2009;16:977990.

- 11 Bruzoni M, Parikh P, Celis R, Are C, Quan PL, Meza JL, Sasson AR: Management of the primary tumor in the patients with metastatic pancreatic neuroendocrine tumor: a contemporary single-institution review. Am J Surg 2009;197:376-381.

12 DerSimonian R, Laird N: Meta-analysis in clinical trials. Control Clin Trials 1986;7: 177-188.
13 Higgins JPT, Thompson SG: Quantifying heterogeneity in a meta-analysis. Stat Med 2002;21:1539-1558.

14 Egger M, Davey Smith G, Schneider M, Minder C: Bias in meta-analysis detected by a simple, graphical test. BMJ 1997;315:629634.

15 Bettini R, Mantovani W, Boninsegna L, Crippa S, Capelli P, Bassi C, Scarpa A, Pederzoli P, Falconi M: Primary tumour resection in metastatic nonfunctioning pancreatic endocrine carcinomas. Dig Liver Dis 2009;41:49-55.

16 Schurr PG, Strate T, Rese K, Kaifi JT, Reichelt U, Petri S, Kleinhans H, Yekebas EF, Izbicki JR: Aggressive surgery improves long-term survival in neuroendocrine pancreatic tumors. An institutional experience. Ann Surg 2007;245:273-281.

17 Fischer L, Kleeff J, Esposito I, Hinz U, Zimmermann A, Friess H, Büchler MW: Clinical outcome and long-term survival in 118 consecutive patients with neuroendocrine tumours of the pancreas. Br J Surg 2008;95: 627-635.

18 Nguyen SQ, Angel LP, Divino CM, Schluender S, Warner RR: Surgery in malignant pancreatic neuroendocrine tumors. J Surg Oncol 2007;96:397-403.

19 Solorzano CC, Lee JE, Pisters PW, Vauthey JN, Ayers JD, Jean ME, Gagel RF, Ajani JA, Wolff RA, Evans DB: Nonfunctioning islet cell carcinoma of the pancreas: survival results in a contemporary series of 163 patients. Surgery 2001;130:1078-1085.

20 Madeira I, Voss TM, Denys A, Sauvanet A, Flejou JF, Vilgrain V, Belghiti J, Bernades P, Ruszniewski P: Prognostic factors in patients with endocrine tumors of the duodenopancreatic area. Gut 1998;43:422-427.

21 Pape UF, Berndt U, Müller-Nordhorn J, Böhmig M, Roll S, Koch M, Willich SN, Wiedenmann B: Prognostic factors of long-term outcome in gastroenteropancreatic neuroendocrine tumours. Endocr Relat Cancer 2008; 15:1083-1097.

22 Eriksson B, Klöppel G, Krenning E, Ahlman $\mathrm{H}$, Plöckinger U, Wiedenmann B, Arnold R, Auernhammer C, Körner M, Rindi G, Wildi S, Frascati Consensus Conference Participants: Consensus guidelines for the management of patients with digestive neuroendocrine tumors - well-differentiated jejunalileal tumor/carcinoma. Neuroendocrinology 2008;87:8-19.
23 Falconi M, Plockinger U, Kwekkeboom DJ, Manfredi R, Korner M, Kvols L, Pape UF, Ricke J, Goretzki PE, Wildi S, Steinmuller T, Oberg K, Scoazec JY, Frascati Consensus Conference; European Neuroendocrine Tumor Society: Well- differentiated pancreatic nonfunctioning tumours/carcinoma. Neuroendocrinology 2006;84:196-211.

24 Hellman P, Lundström T, Ohrvall U, Eriksson B, Skogseid B, Oberg K, Tiensuu Janson E, Akerström G: Effect of surgery on outcome of midgut carcinoid disease with lymph node and liver metastases. World J Surg 2002;26:991-997.

25 Ahmed A, Turner G, King B, Jones L, Culliford D, McCance D, Ardill J, Johnston BT, Poston G, Rees M, Buxton-Thomas M, Caplin M, Ramage JK: Midgut neuroendocrine tumours with liver metastases: results of the UKINETS study. Endocr Relat Cancer 2009; 16:885-894.

26 Gurusamy KS, Ramamoorthy R, Sharma D, Davidson BR: Liver resection versus other treatments for neuroendocrine tumours in patients with resectable liver metastases. Cochrane Database Syst Rev 2009;2:CD007060.

27 Gurusamy KS, Pamecha V, Sharma D, Davidson BR: Palliative cytoreductive surgery versus other palliative treatments in patients with unresectable liver metastases from gastro-entero-pancreatic neuroendocrine tumours. Cochrane Database Syst Rev 2009; 1:CD007118.

28 Frilling A, Li J, Malamutmann E, Schmid KW, Bockisch A, Broelsch CE: Treatment of liver metastases from neuroendocrine tumours in relation to the extent of hepatic disease. Br J Surg 2009;96:175-184.

-29 Scarpa A, Mantovani W, Capelli P, Beghelli S, Boninsegna L, Bettini R, Panzuto F, Pederzoli P, Delle Fave G, Falconi M: Pancreatic endocrine tumors: improved TNM staging and histopathological grading permit a clinically efficient prognostic stratification of patients. Mod Pathol 2010;23:824-833. 